\title{
Design of a multi-epitope-based peptide vaccine against the $S$ and $N$ proteins of SARS-COV-2 using immunoinformatics approach
}

\author{
Arian Karimi Rouzbahani ${ }^{1}$, Farnaz Kheirandish ${ }^{2,3}$ and Seyedeh Zeinab Hosseini ${ }^{4^{*}}$ (D)
}

\begin{abstract}
Background: As the new pandemic created by COVID-19 virus created the need of rapid acquisition of a suitable vaccine against SARS-CoV-2 to develop Immunity and to reduce the mortality, the aim of this study was to identify SARS-CoV-2 $S$ protein and $\mathrm{N}$ antigenic epitopes by using immunoinformatic methods to design a vaccine against SARS-CoV-2, for which S and N protein-dependent epitopes are predicted. B cell, CTL and HTL were determined based on antigenicity, allergenicity and toxicity that were non-allergenic, non-toxic, and antigenic and were selected for the design of a multi-epitope vaccine structure. Then, in order to increase the safety of $\mathrm{Hbd}-3$ and $\mathrm{Hbd}-2$ as adjuvants, they were connected to the $\mathrm{N}$ and $\mathrm{C}$ terminals of the vaccine construct, respectively, with a linker. The three-dimensional structure of the structure was predicted and optimized, and its quality was evaluated. The vaccine construct was ligated to $\mathrm{MHCl}$. Finally, after optimizing the codon to increase expression in E. coli $\mathrm{K} 12$, the vaccine construct was cloned into pET28a $(+)$ vector.

Results: Epitopes which were used in our survey were based on non-allergenic, non-toxic and antigenic. Therefore, 543-amino-acid-long multi-epitope vaccine formation was invented through linking 9 cytotoxic CTL, 5 HTL and 14 B cell epitopes with appropriate adjuvants and connectors that can control the SARS coronavirus 2 infection and could be more assessed in medical scientific researches.
\end{abstract}

Conclusion: We believe that the proposed multi-epitope vaccine can effectively evoke an immune response toward SARS-COV-2.

Keywords: SARS-CoV-2, Multi-epitope, Vaccine, Immunoinformatics, Antigenicity

\section{Background}

Since the advent of SARS-CoV in 2002 and its spread to 32 countries, the world has experienced the outbreak of MERS-CoV and now nCoV 2019 [1]. Coronavirus 2019 (COVID-19), caused by SARS-CoV-2, was first reported in a number of patients with pneumonia of unknown etiology in Hubei Province, China, and subsequently in

\footnotetext{
${ }^{*}$ Correspondence: hosseini.z@lums.ac.ir

${ }^{4}$ Razi Herbal Medicines Research Center, Lorestan University of Medical

Sciences, Khorramabad, Iran

Full list of author information is available at the end of the article
}

many parts of the world [2]. Coronaviruses have four genera: alpha, beta, delta and gamma virus. SARS-CoV-2 belongs to the beta-coronavirus genus with an envelope with a single-stranded RNA genome, positive sense, and has a diameter of about $80-120 \mathrm{~nm}[3,4]$. Their genome size is about 26-32 kilobases [4]. Coronaviruses can infect humans and other vertebrates and cause infections in the respiratory system, gastrointestinal tract and central nervous system of humans, livestock, birds, bats and mice and many other wild animals [5]. SARS-CoV-2, like other coronaviruses, encodes several structural proteins. The structural proteins of SARS-CoV-2 include 
nucleoprotein $(\mathrm{N})$, membrane $(\mathrm{M})$, surface glycoprotein (S) and envelop protein (E) [4]. Most coronaviruses require structural protein to produce a complete viral particle [6]. Each of these proteins is not only involved in the structure of the virus but also in various aspects, involved in virus replication [7]. Surface glycoprotein (S) is responsible for binding to the cellular receptor [8], which has two basic components (S1) and globular (S2). S1 is responsible for binding to the cellular receptor, and S2 contains fusion peptide [9]. For SARS-COV, full-length and the active immunization part of $S$ protein [10], S protein peptides [11] and chimeric versions of $S$ protein have been identified [12]. DNA structures encoding the $\mathrm{S}$ protein have also produced virus-neutralizing antibodies [13]. S protein as a major antigenic component is an important target for vaccine development [14]. Nucleoprotein $(\mathrm{N})$ is a phosphoprotein and nucleocapsid protein that binds to genomic RNA and M protein and is the main stimulus of the host immune system during viral infection [15]. N protein in its entirety is highly immunogenic and antigenic [16]. In addition, $\mathrm{N}$ protein is an early diagnostic marker for SARS-COV because it can be detected in clinical specimens one day after the onset of symptoms [17] and it is stable due to very small mutations [18]. Although ritonavir and lopinavir are used as protease-inhibiting drugs for the treatment of SARS$\mathrm{COV}-2$, it has been reported in a clinical trial that its usefulness for the treatment of SARS-COV-2 is questionable [19]. In case of emergency, Remdesivir is used against SARS-COV-2 or recovered patients' plasma is used as a side-effect-free treatment [20]. However, there is no specific and approved drug for SARS-COV-2 infection, and the treatment approach is more supportive, and the use of these therapies is said to reduce the resulting mortality rate. Therefore, the development of effective drugs and vaccines against the control of emerging diseases is a priority of research and immunoinformatic is currently considered as a new method to find an effective way to control diseases [21]. Immunoinformatics methods could be used to explore antigens of viruses, prediction of their epitopes and evaluation of its immunogenicity [22]. In different studies, medical procedures against the Middle East respiratory syndrome coronavirus (MERS-CoV), Zika virus and Ebola virus were performed by utilizing immunoinformatics techniques [23, 24].

The use of epitope vaccines using immunogenic epitopes specific to CD8+ and CD4+ cells and stimulating the immune system against these epitopes simultaneously and completely specifically are among the methods that have been considered in this regard. Conventional methods for producing vaccines are time-consuming and expensive [25]. The immune system can respond to any viral or microbial contamination, by detecting foreign intruders through their artificial peptide epitopes. By having a total map of virus epitopes and their immunogenicity, it is vital to create an effective vaccine against COVID-19 virus disease [26]. Moreover, multi-epitope vaccine significantly stimulates humoral and cellular immune responses, concurrently due to $\mathrm{T}$ cell as well as B cell epitopes [27, 28]. Multi-epitope vaccine is made of adjuvants, so they are expected to create long-standing immune reactions and high immunogenicity [28].

The aim of this study was to evaluate T-cell- and B-celldependent epitopes derived from SARS-CoV-2 $\mathrm{S}$ and $\mathrm{N}$ antigens for the design and development of a multiepitope vaccine based on the analysis of immunoinformatic tools.

\section{Methods}

\section{Sequence extraction and protein structure}

The FASTA sequences of S protein (YP_009724390.1) and N protein (YP_009724397.2) SARA-COV-2 were retrieved from the NCBI GenBank database (https://ww. ncbi.nlm.nih.gov/) and also Human $\beta$-defensin-2 (PDB ID: 1FD3) and 3 (PDB ID: 1KJ6) from PDB database (https://www.rcsb.org).

\section{Prediction of B cell immune epitopes}

An antigen must be able to elicit both the $B$ and $T$ cell immune responses in order to be a suitable candidate for the vaccine. Therefore, for predicting $B$ cell linear epitopes two servers were used, IEDB (https://www.iedb. org/) and ABCPred (http://crdd.osdd.net/raghava/abcpr ed/ABC_submission.html). ABCPred ranks epitopes by using ANN scores, according to the score obtained and above the threshold (0.5); thus, it is more probable for a sequence to be an epitope with a higher score. For predicting linear epitopes with ABCPred server, 16 mer length of epitopes with default threshold (0.51) and for IEDB server with BepiPred linear epitope prediction method which predicts the location of B cell linear epitopes using a combination of a hidden Markov model and an orientation degree method were selected by default [29]. From matching the predicted linear epitopes with the two servers of IEDB and ABCPred, the epitopes with the highest overlap were selected for further study.

\section{Prediction of CTL and HTL epitopes}

In order to predict CTL epitopes, ComPred method (combination of artificial neural network method and quantitative matrix) was used along with default cutoff score (0.5) of nHLAPred server (https://webs.iiitd.edu. in/raghava/nhlapred.comp.html). 0.18 epitopes were selected with the highest score of alleles and with the highest frequency of Iranian population (HLA * A02:01) and (HLA * B35:01) according to the server (http://allel 
efrequencies.net/hla6006a.asp) for analyzing the next ones. HTL epitopes for DRB1 * 0101, DRB1 * 1101 and DRB1 * 1501 alleles (alleles with the highest frequency from the population of Iran) from NetMHCIIpan 4.0 server (http://www.cbs.dtu.dk/services/NetMHC) were determined [30]. The default threshold was considered for strong connections (rank 0.5\%), weak connections (rank 2\%) and prediction epitope of 15 amino acids long. Predicted epitopes with strong connections were used for further studies.

\section{Evaluation of B cell, CTL and HTL epitopes based on allergenicity, antigenicity and toxicity parameters}

Since the components of the vaccine must be capable of allergic reactions, the selected epitopes for B cell, CTL and HTL with the AllerTOP server v. 2.0 (https://www. ddg-pharmfac.net/AllerTOP/index.html) were reviewed to ensure the ability of selected epitopes to induce an immune response with the VaxiJen v. 2.0 server (http:// www.ddg-pharmfac.net/vaxijen/VaxiJen/VaxiJen.html) and were examined with a threshold of 0.4. This is because toxic epitopes can compromise the structure of the vaccine and should be removed. ToxinPred server (https://webs.iiitd.edu.in/raghava/toxinpred/design.php) with SVM method and default server parameters was used to determine toxic epitopes. Finally, antigen, nonallergenic and non-toxic epitopes were selected as possible epitopes for CTL and HTL B cell.

\section{Vaccine structure design}

The epitopes which were chosen in the previous steps for CTL, HTL and B cell, were selected to design the vaccine structure and were connected by AAY, GPGPG and KK linkers, respectively. In order to improve the immune response, hBD- 3 connected to the $\mathrm{N}$ terminal and hBD-2 connected to the $\mathrm{C}$ terminal of the vaccine construct as an adjuvant to the EAAAK linker.

\section{Evaluation of allergenicity, antigenicity, solubility and stereochemical properties of vaccine structures} Allergenicity assessment has the ability to predict the structure of the vaccine in causing allergies and allergic reactions. Accordingly, the Allergen FP 1.0 server (http:// ddg-pharmfac.net/AllergenFP/) [31] was used. Structural antigenicity designed with ANTIGENpro server (http:// imed.med.ucm.es/Tools/antigenic.pl) [32] and VaxiJen v2.0 (http://www.ddg-pharmfac.net/vaxijen/VaxiJ en/VaxiJen.html) [33] was examined. Vaccine construct solubility prediction was performed with SOLpro server (http://scratch.proteomics.ics.uci.edu/) [34]. The ProtParam server (https://web.expasy.org/protparam) [35] was used to predict stereochemical properties.

\section{Predicting the second and third structures}

SOPMA server (https://npsa-prabi.ibcp.fr/cgi-bin/ npsa_automat.pl?page $=/ \mathrm{NPSA} / \mathrm{npsa}$ _sopma.html) and the PSIPRED server (http://bioinf.cs.ucl.ac.uk/psipr ed/) were used to identify the second structure of the vaccine construct, and the phyre 2 server (http://www. sbg.bio.ic.ac.uk/ phyre2/html/page.cgi?id=index), RaptorX server (http://raptorx.uchicago.edu/Conta ctMap/) and I-TASSER (https://zhanglab.ccmb.med. umich.edu/I-TASSER//) were used to predict the third structure of the vaccine.

\section{Energy optimization and validation evaluation of the third structure of the vaccine structure}

To identify and correct the errors of the selected 3D model, the 3D structure was optimized by using the GalaxyRefine server (http://galaxy.seoklab.org/cgibin/submit.cgi?type=REFINE). ProSA, ERRAT and Ramachandran servers in the software (https://servi cesn.mbi.ucla.edu/PROCHECK/) were used to validate the optimized 3D structure [36-39].

\section{In silico cloning optimization of vaccine construct}

The Backtranseq server (https://www.ebi.ac.uk/Tools/ st/emboss_backtranseq/) [40] was used to reverse the sequence translation of the designed vaccine structure, and expression in the host cell will be reduced owing to the lack of codon optimization; therefore, JCat server (http://www.jcat.de/) [41] was used to optimize the translation codon of $E$. coli $\mathrm{K} 12$ to optimize the codon structure of the multi-epitope vaccine. Finally, the construct sequence of the optimized multi-epitope vaccine was cloned into the pET28a (+) vector using The SnapGene program. Virtual agarose gel simulation was used to virtualize the clone.

\section{Molecular docking}

Server ClusPro 2.0 (https://cluspro.org) [42] was used for protein-protein docking between HLA-A02:01 receptor and ligand (designed vaccine construct). This server fulfilled the task in triple continuous steps like rigid body docking, clustering of lowest form of energy and structural refinement by energy minimization [43]. The best-docked complex was picked according to the minimum energy scoring and docking effectiveness.

\section{Molecular dynamics simulation}

Molecular dynamics is a computational method that was conducted to demonstrate the behavior of molecules and to evaluate the stability of protein-protein complexes [44]. In this study, iMODS server was used to explore the interactivity of the created vaccine and 
its receptor as it has the merit of rapidness and high efficacy [45]. This server evaluates the trend and span of the basic movements of the protein-ligand compound through assessing four prominent reasons: B-factors, eigenvalues, deformability and covariance. In general, when there is high eigenvalue, distortion is very harder [46].

\section{In silico evaluation of immune response}

To evaluate the immunogenicity of the ultimate vaccine, in silico immune simulations were performed by utilizing the C-ImmSim server. This immune trigger applies a position-specific scoring matrix (PSSM) and machine learning methods in order to estimate epitope prediction and immune interactivities, respectively [47].

Clinically, the minimum period of time suggested between two doses of vaccines is 1 month [48]. Immune simulation was conducted by applying the identical protocol reported by previous studies $[49,50]$. In brief, three inoculations were administered with the suggested periods of time of 1 month $(1,84$ and 168 time steps variables were prepared, as one time step is similar to 8 hours of everyday life) for a total of 1050 steps of triggering. All other triggering parameters were kept as defaults.

\section{Results}

Prediction of B cell immune epitopes

The overlap results of the predicted linear $B$ cell epitopes which were found by IEDB and ABCPred servers for proteins $\mathrm{S}$ and $\mathrm{N}$ are shown in Table 1.

\section{Prediction of cytotoxic T lymphocyte and HTL epitopes}

The prediction results of CTL epitopes $(9$ mer) with nHLAPred server (Table 2) and HTL epitopes (15 mer) with NetMHCIIpan 4.0 server for both $\mathrm{S}$ and $\mathrm{N}$ proteins are shown in Table 3.

\section{Selection of epitopes and design of vaccine structures}

The accuracy of the epitopes predicted for B cell, CTL and HTL, in terms of allergenicity, antigenicity and toxicity, was examined. In order to design the vaccine structure, both non-allergenic and non-toxic epitopes that had antigenic potency were selected. Finally, 9 CTL epitopes (5 epitopes for $\mathrm{N}$ protein and 4 epitopes for $\mathrm{S}$ protein), $5 \mathrm{HTL}$ epitopes (1 epitope for $\mathrm{N}$ protein, 4 epitopes for $\mathrm{S}$ protein (Table 4 ) and $14 \mathrm{~B}$ cell epitopes (11 epitopes for $\mathrm{N}$ protein and 3 epitopes for $S$ protein) (Table 5) were selected for vaccine structure design. The selected CTL, HTL and B cell epitopes were
Table 1 Overlap results of predicted linear B cell epitopes of $I E D B$ and $A B C$ Pred servers of $S$ and $N$ proteins

\begin{tabular}{|c|c|c|c|}
\hline \multicolumn{2}{|l|}{$S$ protein } & \multicolumn{2}{|l|}{$\mathrm{N}$ protein } \\
\hline Position & Peptide sequence & Position & Peptide sequence \\
\hline 329 & FPNITNLCPFGEVFNA & 249 & KSAAEASKKPRQKRTA \\
\hline 374 & FASVYAWNRKRISNCV & 24 & TGSNQNGERSGARSKQ \\
\hline 674 & YQTQTNSPRRARSVAS & 59 & HGKEDLKFPRGQGVPI \\
\hline 1153 & DKYFKNHTSPDVDLGD & 182 & ASSRSSSRSRNSSRNS \\
\hline 19 & TTRTQLPPAYTNSFTR & 376 & ADETQALPQRQKKQQT \\
\hline 1139 & DPLQPELDSFKEELDK & 77 & NSSPDDQIGYYRRATR \\
\hline 374 & FSTFKCYGVSPTKLND & 362 & TFPPTEPKKDKKKKAD \\
\hline 1146 & DSFKEELDKYFKNHTS & 243 & GQTVTKKSAAEASKKP \\
\hline 250 & TPGDSSSGWTA & 237 & KGQQQQGQTVTKKSAA \\
\hline 786 & KQIYKTPPIKDFGGF & 32 & RSGARSKQRRPQGLPN \\
\hline 659 & YTMSLGAENSVAYSNN & 169 & KGFYAEGSRGGSQASS \\
\hline 484 & EGFNCYFPLQSYGFQP & 71 & GVPINTNSSPDDQIGY \\
\hline 445 & VGGNYNYLYRLFRKSN & 200 & GSSRGTSPARMAGNGG \\
\hline 457 & RKSNLKPFERDISTEI & 387 & KKQQTVTLLPAADLDD \\
\hline 177 & MDLEGKQGNFKNL & 143 & KDHIGTRNPANNAAIV \\
\hline 293 & LDPL & 65 & KFPRGQGVPINTNSSP \\
\hline 555 & SNKKFLPF & 192 & NSSRNSTPGSSRGTSP \\
\hline 580 & QTLE & 266 & RLNQLESKMSGKGQQQ \\
\hline 602 & TNTSN & 4 & NGPQNQRNAPRI \\
\hline 634 & RVYSTGSNVFQ & 119 & AGLPYGANK \\
\hline 695 & YTMSLGAENSVAYSNN & 343 & DPNFKD \\
\hline 773 & EQDKNTQ & 404 & SKQLQQSMSSADS \\
\hline 807 & PDPSKPSK & & \\
\hline 988 & EAEVQ & & \\
\hline 1035 & GQSKRVDFC & & \\
\hline 1107 & RNFYEPQIITTD & & \\
\hline
\end{tabular}

connected by AAY, GPGPG and KK linkers, respectively. As adjuvant, hBD-3 with 45 amino acids and hBD-2 with 41 amino acids were added to the $\mathrm{N}$ and $\mathrm{C}$ ends of the structure with EAAAK linker. The final vaccine construct consisted of 543 amino acids (Fig. 1).

\section{Predicting the next three structures and optimizing the vaccine structure}

Phyre2, RaptorX and I-TASSER were used in order to predict the three-dimensional structure of different servers, which were evaluated by Ramachandran map. After reviewing the characteristics and validity of the predicted structures by using different servers, the structure received from the RaptorX server, which had a better quality than other servers, was selected. GalaxyRefine server was used to optimize the selected 3D structure. Out of five optimization models, model number 4 with higher RMSD and GDT-HA was selected (Fig. 2a and Additional file 1). 
Table 2 Results of CTL S and N protein epitope prediction by nHLApred server

\begin{tabular}{|c|c|c|c|}
\hline \multicolumn{2}{|l|}{ S protein } & \multicolumn{2}{|l|}{$\mathrm{N}$ protein } \\
\hline Position & Peptide sequence & Position & Peptide sequence \\
\hline 6 & VLLPLVSSQ & 222 & LLLDRLNQL \\
\hline 7 & LLPLVSSQC & 316 & GMSRIGMEV \\
\hline 109 & TLDSKTQSL & 331 & LTYTGAIKL \\
\hline 119 & IVNNATNVV & 406 & QLQQSMSSA \\
\hline 151 & SWMESEFRV & 48 & NTASWFTAL \\
\hline 241 & LLALHRSYL & 226 & RLNQLESKM \\
\hline 269 & YLQPRTFLL & 215 & GDAALALLL \\
\hline 502 & GVGYQPYRV & 393 & TLLPAADLD \\
\hline 512 & VLSFELLHA & 138 & ALNTPKDHI \\
\hline 24 & LPPAYTNSF & 45 & LPNNTASWF \\
\hline 56 & LPFFSNVTW & 66 & FPRGQGVPI \\
\hline 84 & LPFNDGVYF & 105 & SPRWYFYYL \\
\hline 208 & TPINLVRDL & 222 & LLLDRLNQL \\
\hline 241 & LLALHRSYL & 278 & GPEQTQGNF \\
\hline 250 & TPGDSSSGW & 395 & LPAADLDDF \\
\hline 321 & QPTESIVRF & 219 & LALLLLDRL \\
\hline 478 & TPCNGVEGF & 312 & SAFFGMSRI \\
\hline 704 & SVAYSNNSI & 111 & YYLGTGPEA \\
\hline
\end{tabular}

\section{Analysis of stereochemical properties and prediction of solubility, allergenicity, toxicity and antigenicity of vaccine structures}

Examination of the stereochemical properties of the designed structure using ProtParam program showed that the molecular weight of the vaccine structure is 59038.88 daltons with an isoelectric point of 10.06 which shows the basic nature of the designed vaccine structure. The total number of negatively charged amino acids (glutamic, aspartic acid) is 39, and the total number of positively charged amino acids (arginine, lysine) is 104. The aliphatic index is 57.24 and the instability of the designed structure was reported to be 38.96 , which indicates the stability of the vaccine structure designed in the host. GRAVY index was -0.7 , and it was reported that the negative of this index indicates that the vaccine structure is hydrophilic, so it could interact well with water molecules. The half-life of this vaccine construct was predicted to be $30 \mathrm{~h}$ in mammals (in vivo), more than $20 \mathrm{~h}$ in yeast (in vivo) and more than $10 \mathrm{~h}$ in E. coli (in vivo). Based on the results of the SOLpro server, the designed vaccine structure was predicted soluble with a probability of 0.9 , which ensures easy access to the host. Also designed vaccine structures were predicted to be nontoxic, non-allergenic and antigenic. The result of its antigenicity according to VaxiJen and ANTIGENpro servers is 0.5 and 0.9 , respectively.
Table 3 HTL epitope prediction results by NetMHCllpan 4.0 server

\begin{tabular}{lllll}
\hline S protein & & & N protein & \\
\cline { 1 - 2 } Position & Peptide sequence & & Position & Peptide sequence \\
\hline 867 & DEMIAQYTSALLAGT & & 388 & KQQTVTLLPAADLDD \\
309 & EKGIYQTSNFRVQPT & & 168 & PKGFYAEGSRGGSQA \\
430 & TGCVIAWNSNNLDSK & & 374 & KDQVILLNKHIDAYK \\
232 & GINITRFQTLLALHR & & 311 & ASAFFGMSRIGMEVT \\
512 & VLSFELLHAPATVCG & & 354 & NKHIDAYKTFPPTEP \\
59 & FSNVTWFHAIHVSGT & & \\
323 & TESIVRFPNITNLCP & & \\
1001 & LQSLQTYYTTQQLIRA & & \\
314 & QTSNFRVQPTESIVR & & \\
790 & KTPPIKDFGGFNFSQ & & \\
978 & NDILSRLDKVEAEVQ & & \\
540 & NFNFNGLTGTGVLTE & & \\
\hline
\end{tabular}

Features of the secondary predicted structure

The second structure of the protein using the PSIPRED program is shown in Fig. 3. Also, according to the results of SOPMA program, the protein has 145 alpha helices (26.70\%), 84 extended strands (15.47\%), $36 \beta$-turn (6.63\%) and 278 random coils (51.20\%) (Additional file 1 ).

\section{Validation of the optimized three-dimensional structure of the vaccine structure}

Structural validation is a procedure to recognize potential flaws in the estimated tertiary structure [51]. The overall quality assessment of the optimized 3D structure was evaluated with ProSA, ERRAT and PROCHECK servers. According to the results of ERRAT and ProSA servers, the quality factor was 92,000 and Z-score of the structure reported was -9.29 (Additional file 1) which is in the range of scores that are normally found for natural proteins of similar size (Fig. 2c). Also, according to the Ramachandran map which was obtained from the PROCHECK server, the number of amino acids in favored and allowed regions is $94.6 \%$ and $5.4 \%$, respectively, and in outlier regions, $0.0 \%$ was reported (Fig. 2b).

\section{Codon optimization and in silico cloning}

In order to evaluate the cloning and expression of the vaccine construct in the expression vector, the inverse translation of the vaccine construct sequence was received by the Backtranseq server and its codon was optimized by the JCAT server. The JCAT server evaluates the sequence to optimize the codon and reports the codon compatibility index (CIA) and GC content of the 


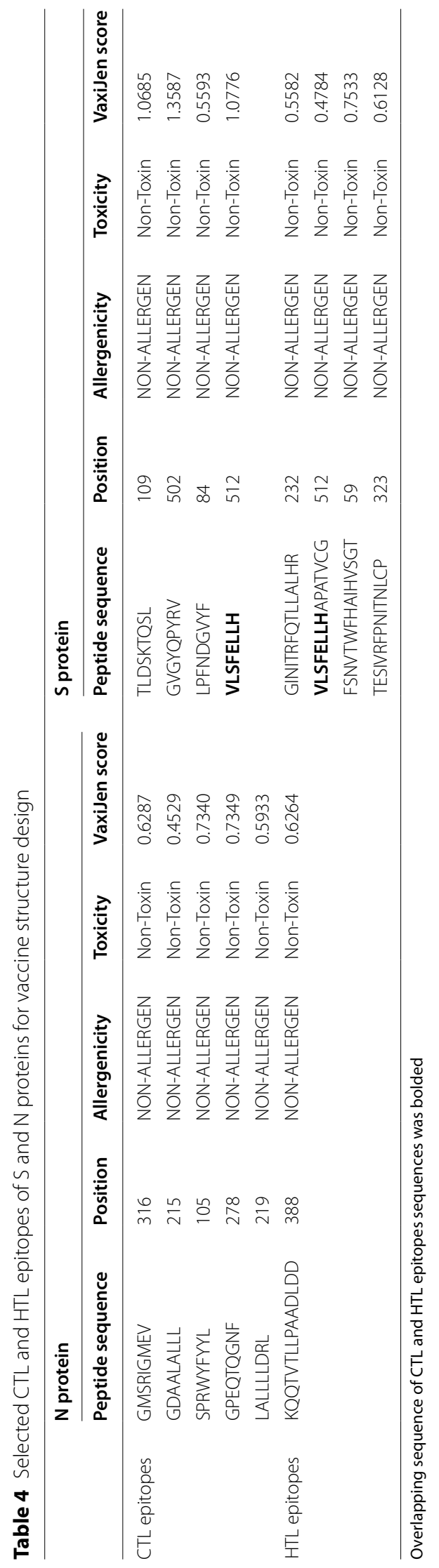




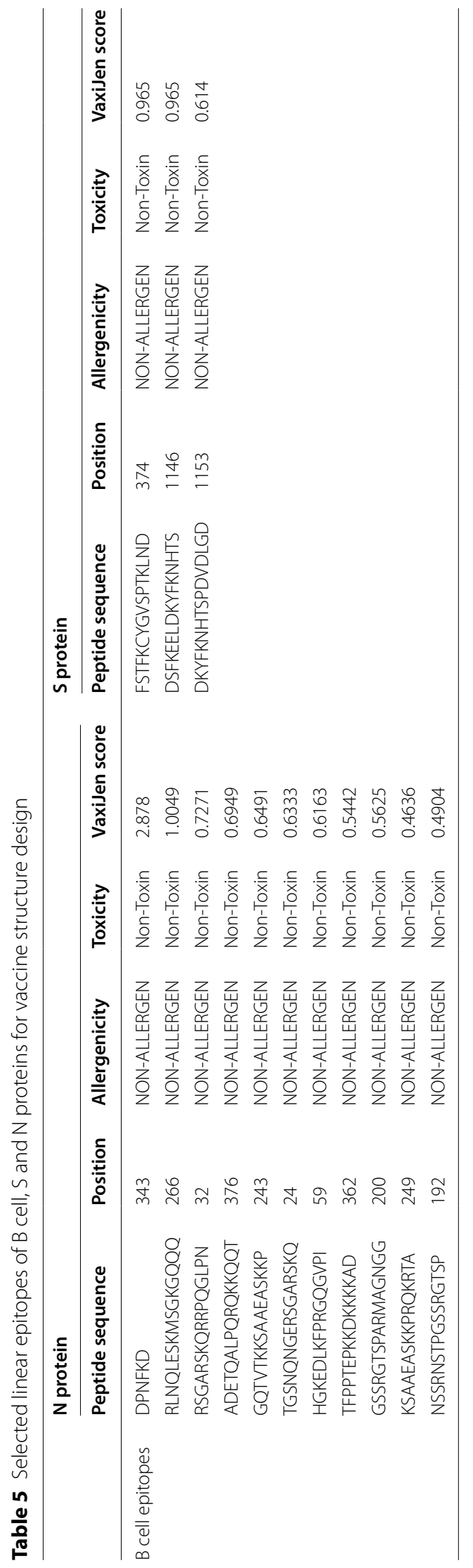




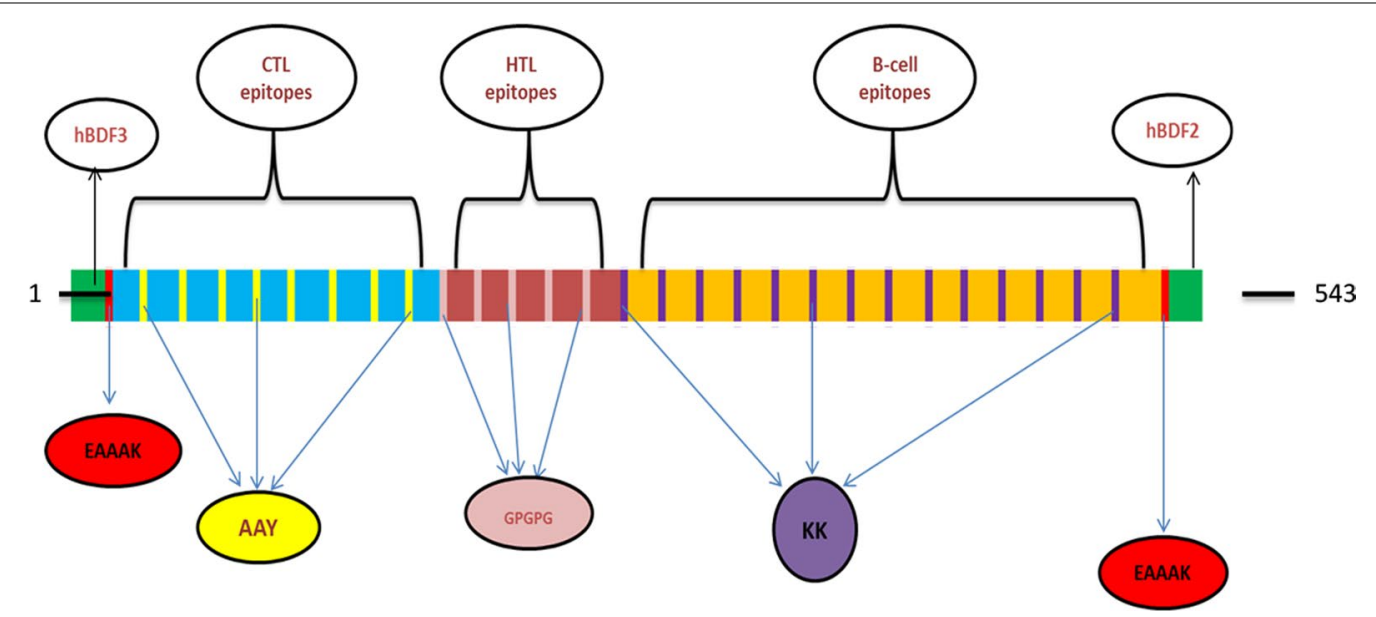

Fig. 1 Schematic specifications of the structure of a 543-amino-acid-long multi-epitope vaccine. Adjuvant with EAAAK linker is added to the beginning and end of the structure, and CTL, HTL and B cell epitopes are connected with AAY, GPGPG and KK linkers, respectively

sequence. According to the results, the codon compatibility index (1.0), which was in the optimal range $(0.8-$ 1.0), was calculated. A high CIA value indicates high gene expression. Also, GC sequence content (51.93\%) was in the desired range (30-70\%). These results may indicate high expression of the vaccine construct in the bacterial system. Finally, after adding the BamHI and XhoI restriction enzymes to the sequence, the optimized codon sequence was cloned using the SnapGene program in the pET28a (+) vector (Fig. 4). The SnapGene program virtual agarose gel simulation shows the presence of insert alone, along with vector after digestion with BamHI and Xhol enzymes (Fig. 5).

\section{Molecular docking}

Docking of the designed vaccine construct as a ligand with HLA-A02;01 (PDB ID: 3TO2) as the receptor was performed by the ClusPro 2.0 server. This server predicts 30 complexes and classifies them based on the amount of energy. Among the predicted models, model 4, which had the lowest energy weighted score of -1158.9 , has been selected as the best model for vaccine interaction with HLA-A02:01 (Fig. 6). Additionally, PDBsum as a virtual database was applied to show the interacting residues of docked complexes [52]. An amount of 46 vaccine residues was matched with 42 residues of chain A from HLA-A02;01 molecule. Also, 25 hydrogen bonds were built between the residues of the chain A from the HLA-A02:01 molecule (Fig. 7).

\section{Molecular dynamics simulation}

To assess the firmness and physical motions of the created vaccine composition-HLA-A02:01 docked compound. Molecular dynamics simulation was performed through the iMOD server [46]. The main chain deformability is displayed in Fig. 8A. The region where hinges are located has a high tendency to deform. The B-factor values computed by normal mode analysis are proportional to root mean square (Fig. 8B). Values of B-factor measure the unpredictability of each atom. Figure $8 \mathrm{C}$ introduces the eigenvalues having close correlation with the energy needed to distort the formation. The eigenvalue of the complex is $3.23 \mathrm{e}^{-08}$. The covariance matrix between the pairs of residues is displayed in Fig. 8D, showing their correlations (red: correlated, white: uncorrelated, blue: anti-correlated). The elastic network model is indicated in Fig. 8E.

\section{In silico evaluation of immune response}

The immunogenic profile of the designed vaccine candidates was attained from C-IMMSIM server. Simulation outcomes depicted that high concentrations of IgM were recognized at the primary response. In both secondary and tertiary responses, the usual elevated levels of immunoglobulin activities (i.e., IgG1 + IgG2, IgM, and IgG + IgM antibodies) were noticeable with associated antigen depletion (Fig. 9A). The elevated levels of simulated B cells and memory B cell formation were seen, which shows a productive long-established immune reaction created by the vaccine structure (Fig. 9B-D). A further high level of reaction was seen in the $\mathrm{T}$ helper and cytotoxic $\mathrm{T}$ cell populations with relative memory establishment which is necessary 


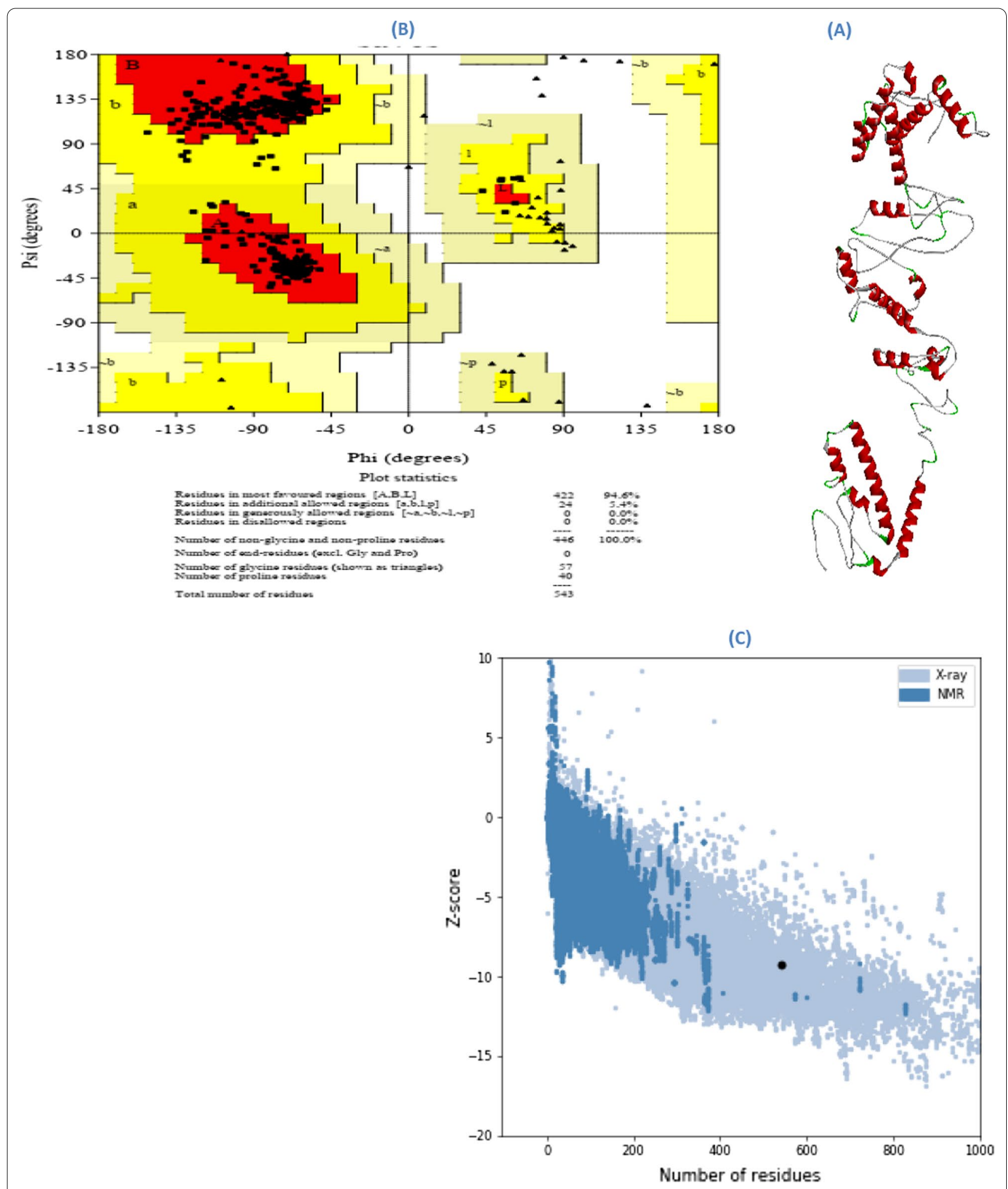

Fig. 2 Evaluation of the third optimized structure of the vaccine structure. a Optimized three-dimensional structure of the vaccine structure, $\mathbf{b}$ analysis of the vaccine structure by Ramachandran map, c Z-score map of ProSA server vaccine structure 
conf cart Pred CHHHHHHHHHEEEECCEEEEEECCCCHHHHCC C C C C CHHHHHHHHHH C AA GI I NTLQKYYCRVRGGRCAVLSCLPKEEQI GKCSTRGRKCCRRKKEAAAK

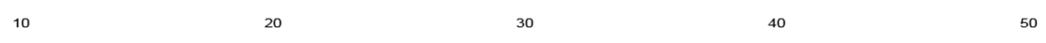

Conf $\square$ प Cart Pred H H H H H H H H C C C C C C C H H H C C C C C H H H H H H H H H H H H H C C C AA LLLDRLAAYTLDSKTQSLAAYGVGYQPYRVAAYVLSFELLHAAAYLPFN 120

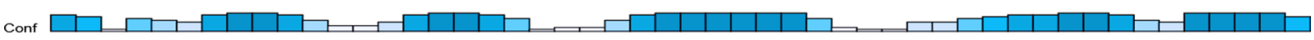

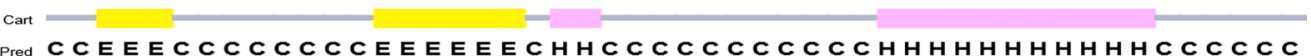
AA DGVYFGPGPGKQQTVTLLPAADLDDGPGPGGINITRFQTLLALHRGPGPG 160 170 180

190

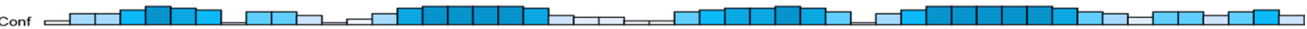
Cart

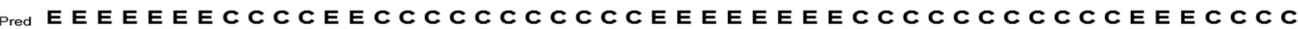
AA VLSFELLHAPATVCGGPGPGFSNVTWFHAI HVSGTGPGPGTESIVRFPNI

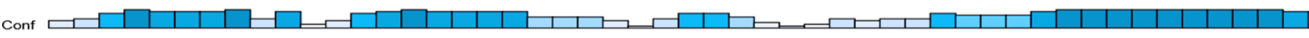
Cart Pred C C C C C C C C C н н н н н н н н н н н н T NLCPKKDPNFKDKKRLNQLESKMSGKGQQQKKRSGARSKQRRPQGLPNK

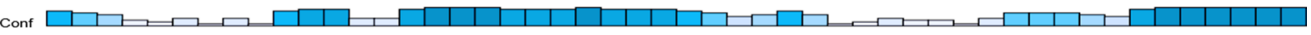
Cart Pred C C C CEECCHH H H C C C C C C C C C C C C C C C H H C C C C C C C C C C C C AA KGQTVTKKSAAEASKKPKKTGSNQNGERSGARSKQKKHGKEDLKFPRGQG 310

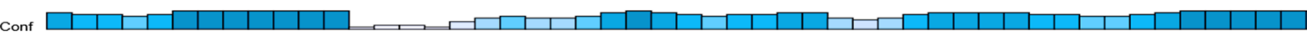
Cart Pred CCCCCCCCCCCCCCCCCCCCCCCCCCCCCCCCCCCCCCCCCCCCCCCCCC APIKKTFPPTEPKKDKKKKADKKGSSRGTSPARMAGNGGKKNSSRNSTP
360
370
380
390
400

Conf (ats Pred $\mathrm{C} C \mathrm{C}$ C C C C C H H H H H C C C H H H C C C C C н H H H H H C C C C C C C C C C C C CE AA SSRGTSPKKADETQALPQRQKKQQTKKKSAAEASKKPRQKRTAKKFSTFK 410 420 430 440 450

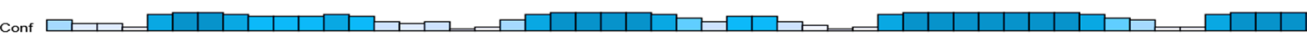
Cart

Pred EE ECC C C C C C C C C C H H H H C C C C C C C C C C C H H H H H H H H H H H H H H AA CYGVSPTKLNDKKDKYFKNHTSPDVDLGDKKDSFKEELDKYFKNHTSEAA 460 470 480 490

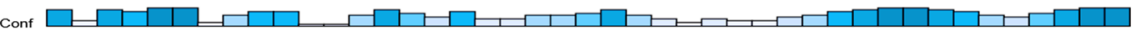
Cart $\mathrm{HCCCCCCHHHCCCCCCCCCEECCCCCCEECCCCCCCCCCCCCC}$ AA AKGI GDPVTCLKSGAICHPVFCPRRYKQIGTCGLPGTKCCKKP

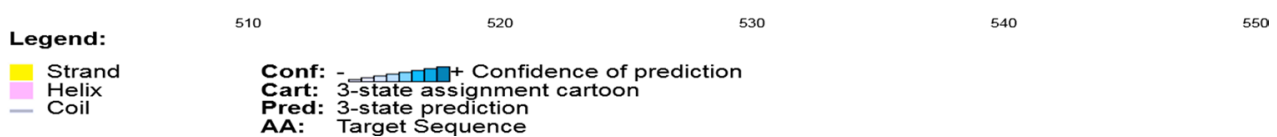

Fig. 3 Two-dimensional analysis of the structure of the vaccine designed by the PSIPRED server 


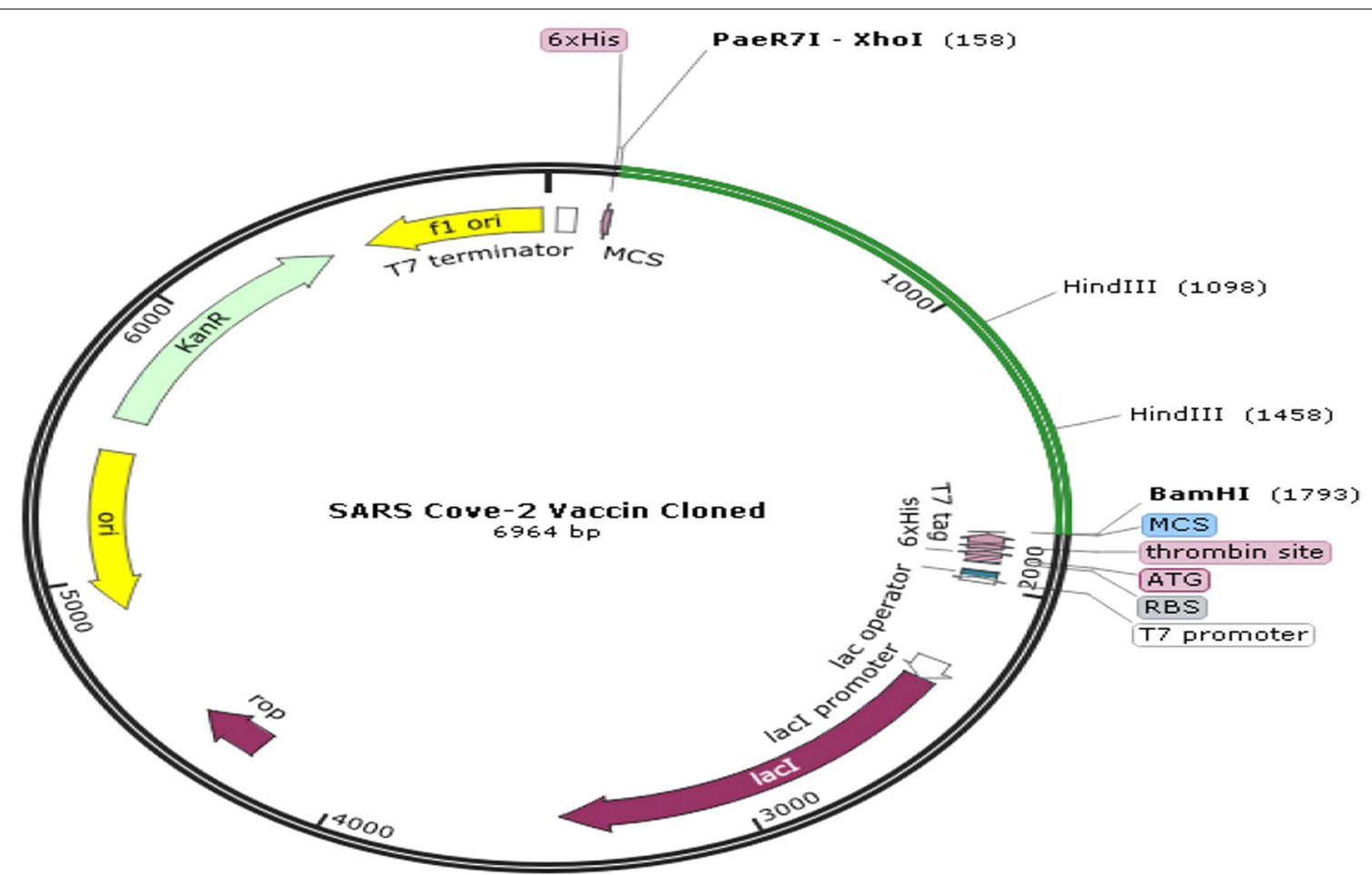

Fig. 4 Clone of the designed vaccine construct. The optimized codon sequence of the designed vaccine construct (shown in green) was cloned between the Xhol and BamHI enzyme loci in the expression vector pET-28a ( + ) (shown in black)

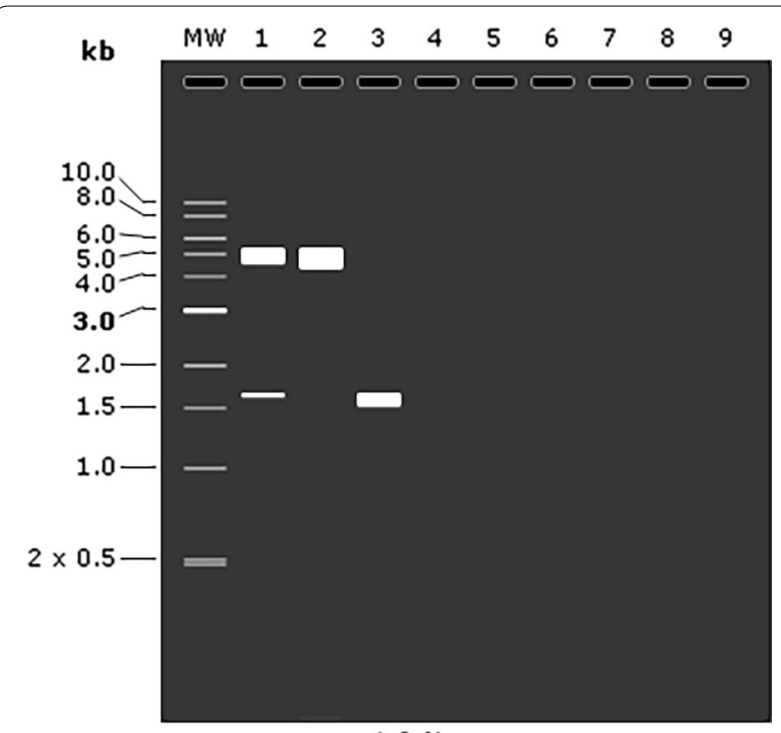

$1.0 \%$ agarose

Fig. 5 Virtual clone of a vaccine construct designed with dual digestion. Line1: Digestive structure of vaccine (vaccine and vector) with two enzymes Xhol and BamHI; line2: digestion of two enzymes, vector pET-28a (+); line 3: digestion of two enzymes, incert (designed vaccine)

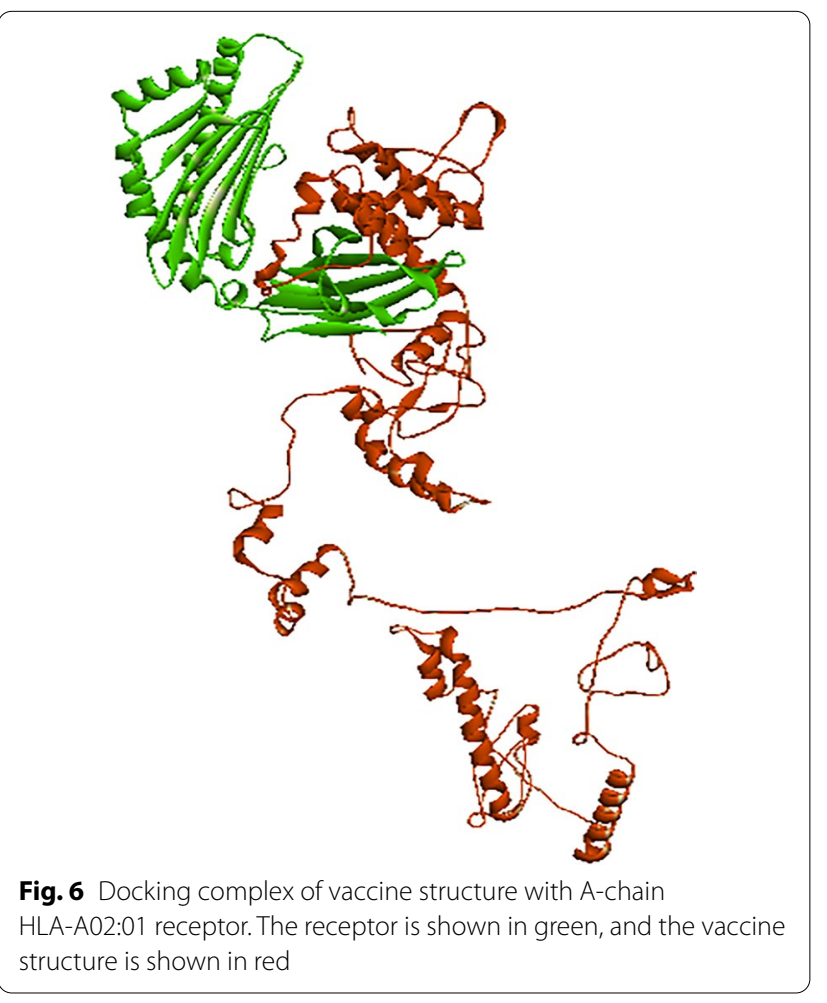




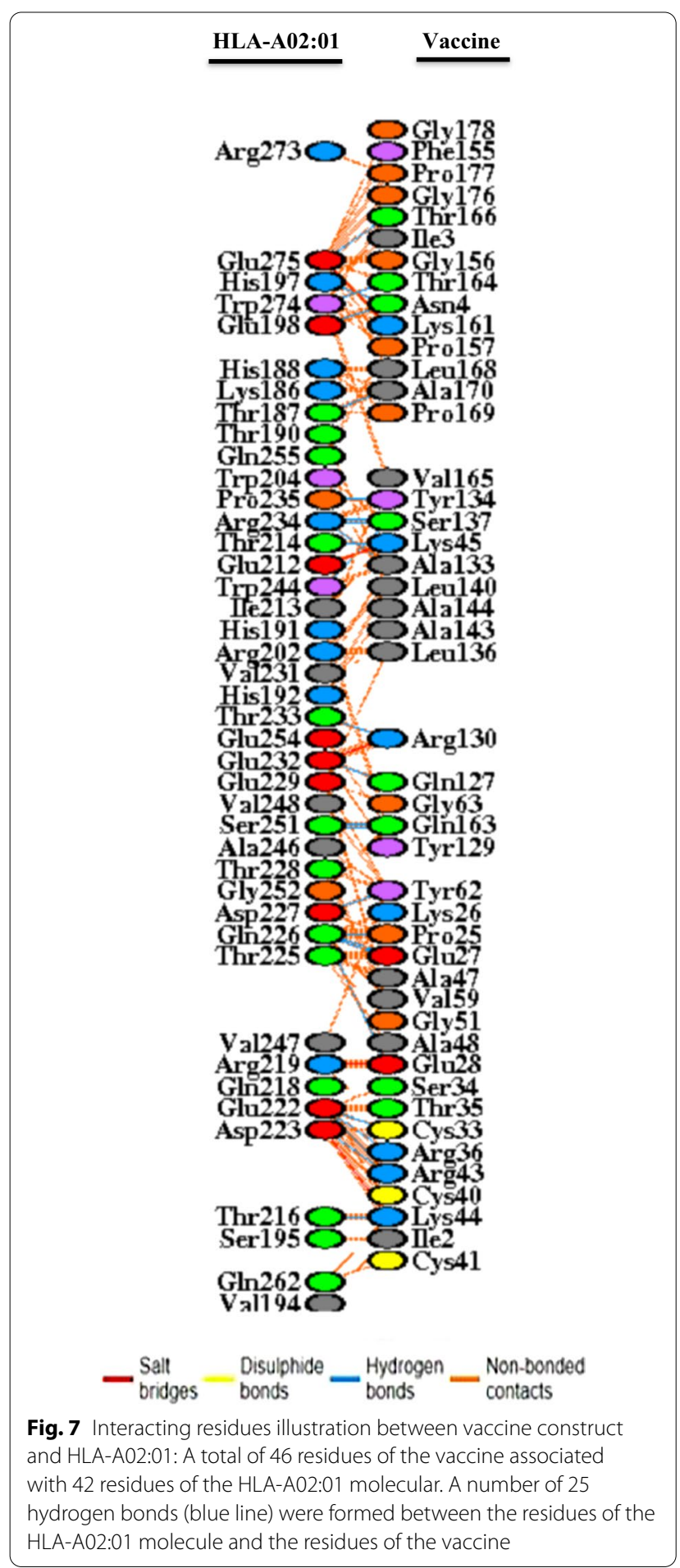

to trigger the immune reaction (Fig. 9E-H). Thus, improved activity of macrophage was observed while dendritic cell activity was steady (Fig. 9I, J). It was also found high level of cytokines including IFN- $\gamma$ and IL-2, which are imperant for inhibition of viral replication and cellular immunity (Fig. 9K). The above-observed immune elicit characteristics ensured that vaccine structure would be effectual in human subjects.

\section{Discussion}

Today, as coronaviruses appear periodically and unpredictably and they are spreading rapidly, they are causing serious infectious diseases; they have become a constant threat to human health. This is especially true when there is no vaccine or approved drug to treat coronavirus infection [1]. Many studies are underway to develop an effective vaccine against SARS-CoV-2. Some studies have suggested that the $S$ protein is a promising candidate for the SARS-CoV-2 vaccine because it is involved in the binding, fusion and entry of the virus into the host cell [53]. There are also reports showing that antibodies against $\mathrm{S}$ protein prevent SARS-CoV-2 from entering cells, so it strengthens the use of $\mathrm{S}$ protein as a suitable candidate for the production of SARS-CoV-2 vaccine [54]. Also, $\mathrm{N}$ protein, due to its protected protein sequence, growing knowledge of its genetic biochemistry and very high immunogenicity, can be considered as a suitable candidate for the production of vaccine against COVID-19 disease [55]. Today, the ease of manufacturing industrial peptides as well as their engineering ability has made such vaccines suitable candidates for vaccination. The use of epitope vaccines based on peptide synthesis is one of the new strategies in vaccine research that focuses the immune response on important and valuable epitopes. The use of epitope peptides for vaccination against various organisms such as HIV (human immunodeficiency virus), HBV (hepatitis B virus) and various models of cancer, etc., has been considered [56]. During the present study, epitopes derived from $\mathrm{S}$ and $\mathrm{N}$ proteins SARS-CoV-2 were studied for the design and development of a multi-epitope vaccine using immunoinformatic methods. Identification of antigenic epitopes by the immune system is a key step in the immune response to the pathogen, identifying either epitopes that stimulate $\mathrm{T}$ cells or epitopes that are trapped by B cells and soluble antibodies [57]. In this study, CTL, HTL and B cell epitopes were selected based on antigenetic, allergenicity and toxicity. A restriction of these published studies is the failure to consider the effect of glycosylation, which could shield some of the selected epitopes. The vital role of glycosylation is defined in antigenicity, fusogenic and immunomodulatory activities of the spike protein [58]. About $17 \mathrm{~N}$-glycosylation sites associated with two O-glycosylation sites were found occupied in the spike protein of SARS-CoV-2 [59]. Meanwhile, glycans could impede the recognition of antigens by shielding the residues [60], and protein glycosylation would impact on the efficiency of antigen finding [61]. We circumnavigated most 
A

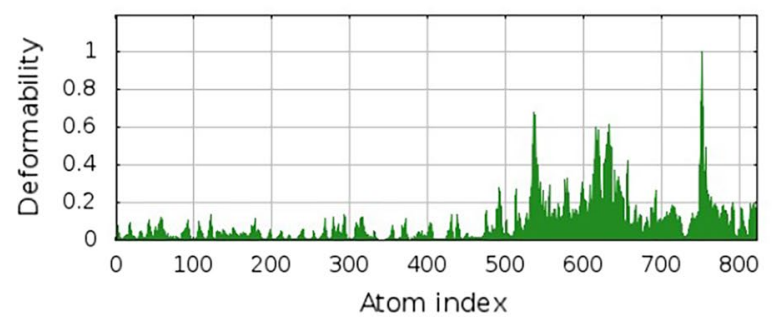

C

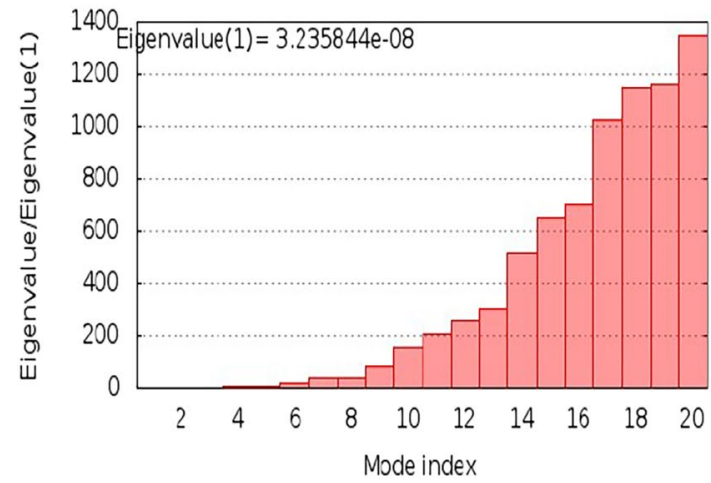

$\mathbf{E}$

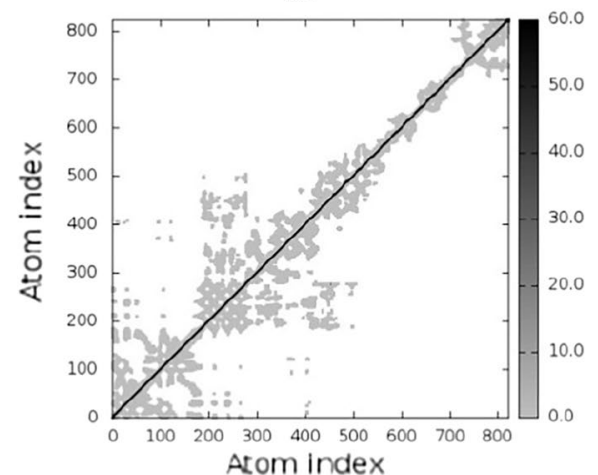

B

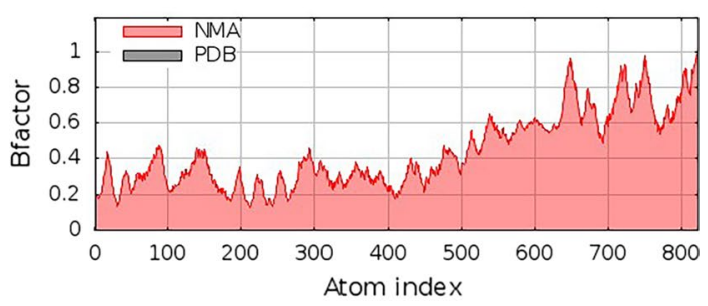

D

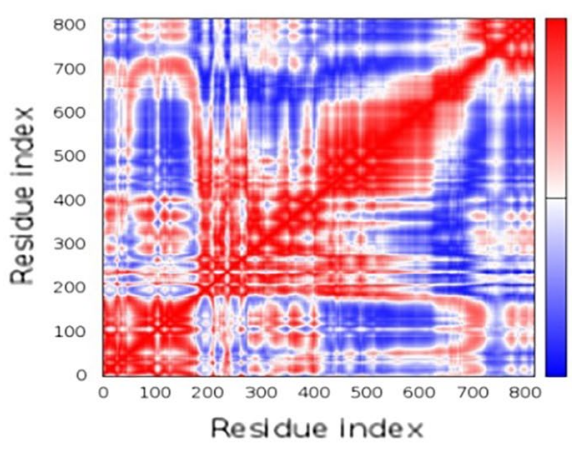

Fig. 8 Molecular dynamics simulation of multi-epitope vaccine-HLA-A02:01 complex; stability of the protein-protein complex was investigated through deformability (A), B-factor values (B), eigenvalue (C), covariance of residue index (D) and elastic network (E) analysis

glycosylation sites when selecting epitopes derived from $S$ protein SARS-CoV-2. In this study, only three selected epitopes (GIN ${ }^{234}$ ITRFQTLLALHR, FSN ${ }^{61}$ VTWFHAIHVSGT, TESIVRFPN ${ }^{331}$ ITNLCP) contain glycosylation sites, which should have a minimum influence on antigen recognition. If these glycosylation sites hinder the diagnostic presentation, an extra deglycosylation step with $N$-glycanase should be useful for the test samples, which is a simple and useful technique for deglycosylation [61]. Many studies have revealed the influence of glycosylation on the augmentation of antigens immunogenicity [62]. Owing to increase expression, folding and stability, linkers act as an essential element in the development of epitope vaccines [63]. In this study, CTL, HTL and B cell epitopes were connected to design vaccine structure by AAY, GPGPG and KK linkers, respectively. Defensins increase the acquired immune response by chemically absorbing activity for monocytes, $\mathrm{T}$ cells and dendritic cells, and the activity of inducing cytokine production by monocytes and epithelial cells [64]. Accordingly, human beta-defensin 3 and 2 were added as adjuvants to the $\mathrm{N}$ and $\mathrm{C}$ ends of the designed structure by the EAAAK linker, respectively. EAAAK linker, due to its salt bridge related to glutamic acid and lysine, can prevent protein which domains from converging by creating a stable helix structure [65]. 
A

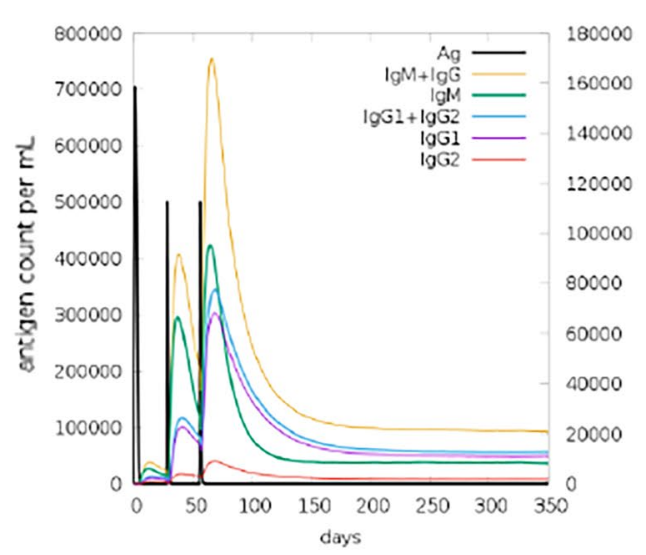

C

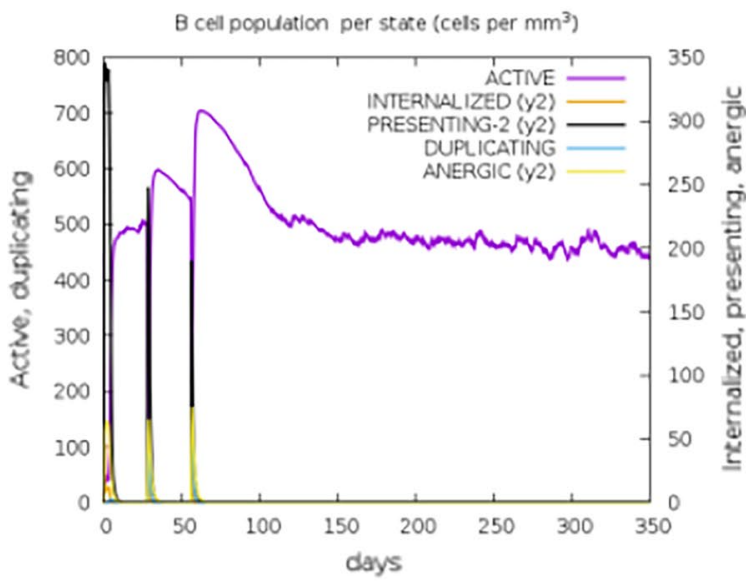

E

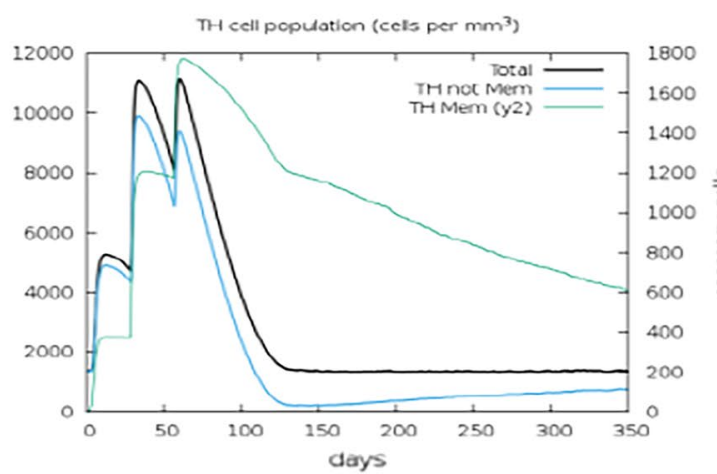

B

B cell popuation (cels per $\mathrm{mm}^{3}$ )

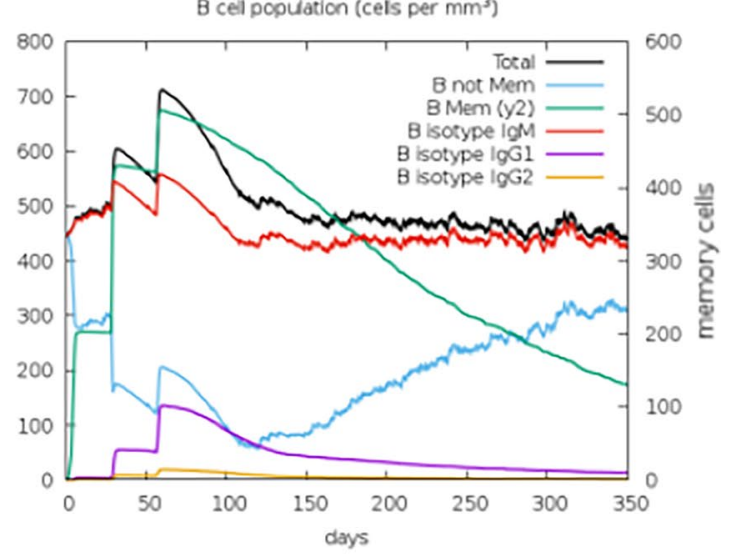

D

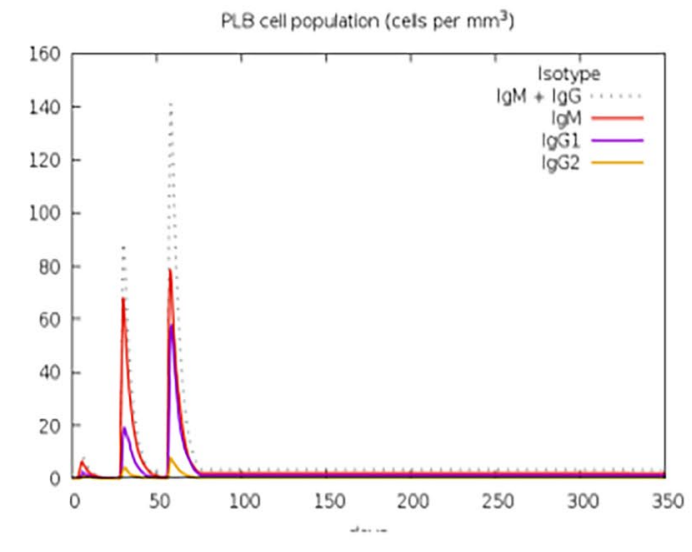

F

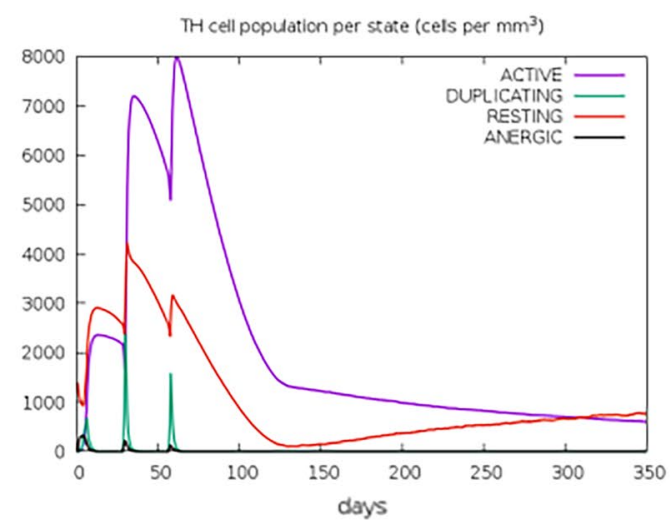

Fig. 9 The immune simulation results of the vaccine construct. A Immunoglobulins levels with respect to antigen concentration, $\mathbf{B} B$ cell population, C B cell population per state, $\mathbf{D}$ plasma B cell population, E helper T cell population, $\mathbf{F}$ helper $\mathbf{T}$ cell population per state, $\mathbf{G}$ cytotoxic $\mathbf{T}$ cell population, $\mathbf{H}$ cytotoxic $\mathbf{T}$ cell population per state, I macrophage population per state, $\mathbf{J}$ dendritic cell population per state and $\mathbf{K}$ production of cytokine and interleukins with Simpson index 


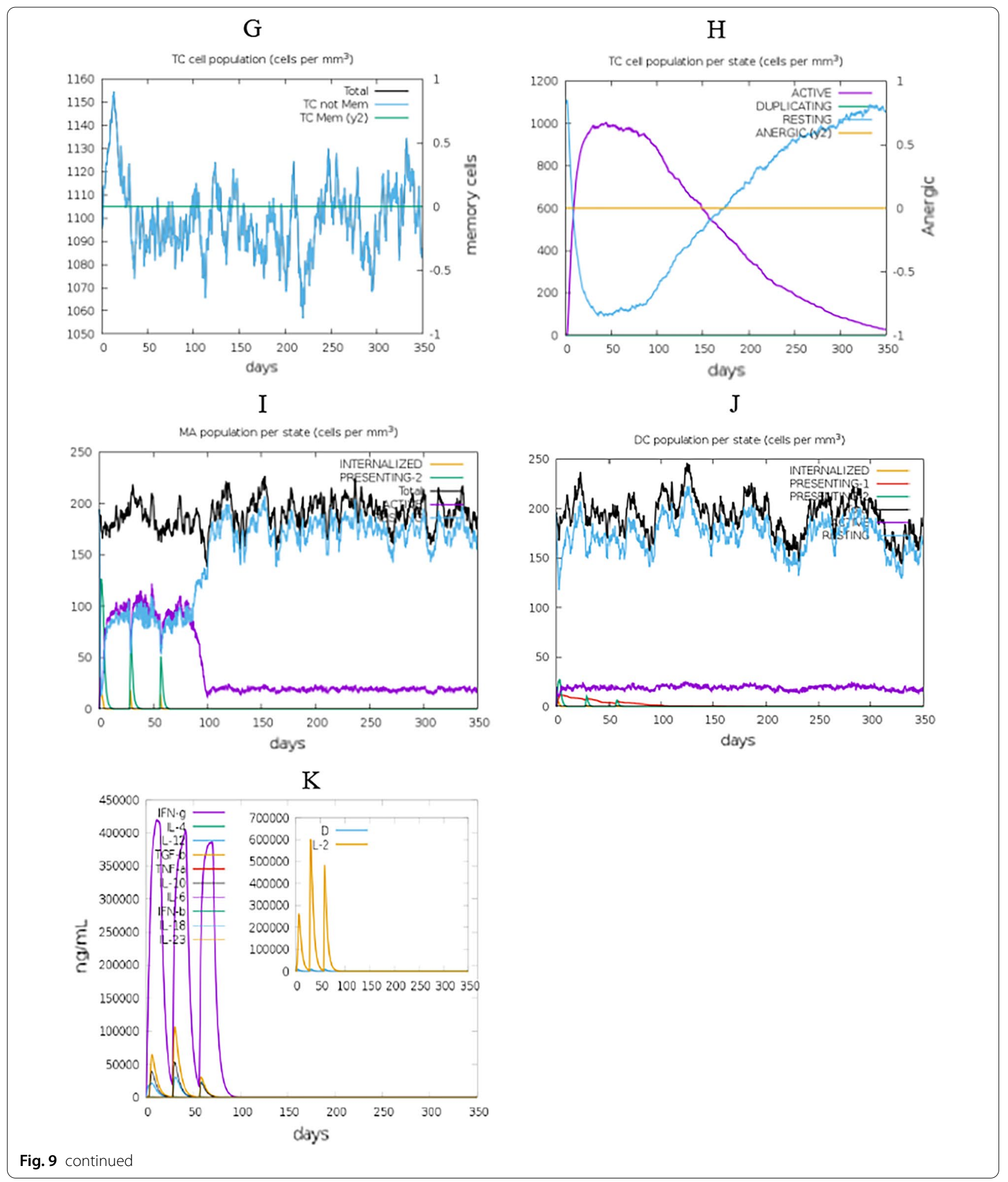

The molecular weight of the designed vaccine was $59,038.88$ daltons (approximately $59 \mathrm{kDa}$ ), which makes it an acceptable vaccine. Because proteins with a molecular weight of less than $110 \mathrm{kDa}$ are considered as more suitable targets for vaccine production [66]. The isoelectric point of the vaccine structure 
was determined to be $10 / 06$, which indicates the playful nature of the designed vaccine structure. Also, the instability index of the structure is 38.96 according to ProtParam program, which is classified as a stable protein. Because the range of this index for stable proteins is less than 40 results, the alpha index, which indicates the stability of the protein over a wide temperature range, was reported to be 57.24 for this designed vaccine construct. Its GRAVY value is -0.7 , which is a negative value of this index, indicating the nature of the hydrophilic structure of the vaccine, and therefore can interact strongly with water molecules. The total number of negatively charged (Asp + Glu) and positive (Arg + Lys) amino acids in this vaccine structure is 39 and 104, respectively. The half-life of this vaccine construct was predicted to be $30 \mathrm{~h}$ in mammals, more than $20 \mathrm{~h}$ in yeast and more than $10 \mathrm{~h}$ in $E$. coli. Based on the results, the structure of the designed vaccine solution was predicted to ensure easy access to the host. Also, according to the predicted results, the structure of the designed vaccine is antigen, non-toxic and non-allergenic. The quality of the three-dimensional structure of the designed vaccine structure increased dramatically after optimization, so that all amino acids in the desired and allowed areas (100\%), according to Ramachandran map, reported that it shows the appropriate quality of the three-dimensional structure of the designed vaccine structure. Various tools were used to determine possible errors and the quality of the three-dimensional structure of the designed vaccine structure. Z-score $(-9.22)$ and ERRAT quality factor $(92,000)$ showed that the structure of the designed vaccine is appropriate. Using ClusPro2.0 server, connection was made between the vaccine structure designed with HLA-A02:01-1158.9 of HLA-A02:01 was the lowest amount of energy in the total of the vaccine structure. Furthermore, the iMODS server was applied to evaluate the constructional steadiness and atomiclevel motions of docked complex (designed vaccine construct-HLA-A02:01). It showed that docked proteins have minor deformation for each residue and with establishing our estimation of eigenvalues for $3.23 \mathrm{e}^{-08}$, which display the validity of our in silico predicted vaccine. Because all codons that are synonymous in a codon family do not use the same rate of expression of heterogeneous proteins in Escherichia coli, codon optimization in production of eukaryotic proteins is necessary in prokaryotic hosts [67]; therefore, codon optimization was performed to achieve a high level of protein expression in E. coli $\mathrm{K} 12$, and according to the results, both codon compatibility index (1.0) and GC percentage $(51.93 \%)$ were calculated; this reveals a high probability of protein expression in bacteria. In addition, the immune simulation of the designed vaccine structure showed hopeful results regarding both humoral and cellular immune reaction. The results of bioinformatics evaluation of the designed vaccine construct indicated that this vaccine candidate may be highly potent against SARS-CoV-2, but in vitro and in vivo studies are needed for clinical confirmation.

\section{Conclusion}

In silico vaccine formation being efficient is substantially important, and it strongly focused on the multiepitope peptides of the vaccine. In this study, using bioinformatics analyses, suitable epitopes of $\mathrm{S}$ and $\mathrm{N}$ proteins were selected and analyzed. Finally, a different multi-epitope vaccine with a span of 543aa against the 2019-nCov will be created.

It consists of two adjuvants, with 14 B cell epitopes, 9 CTL epitopes and 5 HTL epitopes. It displays good antigenic features, immunological qualities and satisfactory physiochemical characteristics, non-allergenicity and non-toxicity. It is expected that the epitopes predicted in this study would be an efficient vaccine formation against COVID-19. However, the confirmation of the epitopes which were selected in this study as a vaccine candidate should be considered as laboratory studies.

\section{Abbreviations}

Hbd2: Human $\beta$-defensin-2; Hbd3: Human $\beta$-defensin-3; 2019-nCoV: 2019 novel coronavirus; SARS-CoV: Severe acute respiratory syndrome coronavirus; MERS: Middle East respiratory syndrome; SARS: Severe acute respiratory syndrome; MHC: Major histocompatibility complex; HLA: Human leukocyte antigen; pl: Isoelectric point; CTL: Cytotoxic T lymphocyte; HTL: Helper T Iymphocyte; COVID-19: Coronavirus disease 2019; GRAVY: Grand average of hydropathy; pl: Isoelectric point; SARS-CoV-2: Severe acute respiratory syndrome coronavirus 2.

\section{Supplementary Information}

The online version contains supplementary material available at https://doi. org/10.1186/s43042-022-00224-w.

Additional file 1. Design of a multi-epitope-based peptide vaccine against the $\mathrm{S}$ and $\mathrm{N}$ proteins of SARS -COV-2 using Immunoinformatics Approach. (http://galaxy.seoklab.org/cgi-bin/report_REFINE.cgi?key= 27ac3cd2f0bd1f0372ce673a67eac9e1, https://npsa-prabi.ibcp.fr/cgi-bin/ secpred_sopma.pl, https://prosa.services.came.sbg.ac.at/prosa.php, https://saves.mbi.ucla.edu/results?job=748446\&p=errat).

\section{Acknowledgements}

The authors appreciate the respected vice-chancellor and colleagues of Research and Technology, Lorestan University of Medical Sciences, for their sincere cooperation.

\section{Authors' contributions}

FKH visualized the study and reviewed and edited the final manuscript. SZH contributed to main analysis and review and editing of the manuscript. AKR helped in main analysis and writing original draft. All authors have read and approved the manuscript. 


\section{Funding}

The authors received no funding for this project from any organization.

\section{Availability of data and materials}

URL links of supplementary files are available in Additional file 1.

\section{Declarations}

\section{Ethics approval and consent to participate}

The present study was approved by The Ethics Committee of Lorestan University of Medical Sciences (IR.LUMS.REC.1399.010).

\section{Consent for publication}

Not applicable.

\section{Competing interests}

The authors declare no competing interest.

\section{Author details}

1 Student Research Committee, Lorestan University of Medical Sciences, Khorramabad, Iran. ${ }^{2}$ Department of Medical Parasitology and Mycology, School of Medicine, Lorestan University of Medical Sciences, Khorramabad, Iran. ${ }^{3}$ Department of Medical Biotechnology, School of Medicine, Lorestan University of Medical Sciences, Khorramabad, Iran. ${ }^{4}$ Razi Herbal Medicines Research Center, Lorestan University of Medical Sciences, Khorramabad, Iran.

Received: 15 July 2021 Accepted: 13 November 2021

Published online: 04 February 2022

\section{References}

1. Li G, Fan Y, Lai Y et al (2020) Coronavirus infections and immune responses. J Med Virol 92(4):424-432

2. Huang C, Wang Y, Li X et al (2020) Clinical features of patients infected with 2019 novel coronavirus in Wuhan, China. Lancet 395(10223):497-506

3. Fung TS, Liu DX (2019) Human coronavirus: host-pathogen interaction. Annu Rev Microbiol 73:529-557

4. Lu R, Zhao X, Li J et al (2020) Genomic characterisation and epidemiology of 2019 novel coronavirus: implications for virus origins and receptor binding. Lancet 395(10224):565-574

5. Ge X-Y, Li J-L, Yang X-L et al (2013) Isolation and characterization of a bat SARS-like coronavirus that uses the ACE2 receptor. Nature 503(7477):535-538

6. Ruch TR, Machamer CE (2012) The coronavirus E protein: assembly and beyond. Viruses 4(3):363-382

7. Kirchdoerfer RN, Cottrell CA, Wang N et al (2016) Pre-fusion structure of a human coronavirus spike protein. Nature 531(7592):118-121

8. Huang Y, Yang C, Xu X, Xu W, Liu S (2020) Structural and functional properties of SARS-CoV-2 spike protein: potential antivirus drug development for COVID-19. Acta Pharmacol Sin 41(9):1141-1149

9. Xia X (2021) Domains and functions of spike protein in Sars-Cov-2 in the context of vaccine design. Viruses 13(1):109

10. He Y, Li J, Du L et al (2006) Identification and characterization of novel neutralizing epitopes in the receptor-binding domain of SARS-CoV spike protein: revealing the critical antigenic determinants in inactivated SARSCoV vaccine. Vaccine 24(26):5498-5508

11. Lien S-P, Shih Y-P, Chen H-W et al (2007) Identification of synthetic vaccine candidates against SARS CoV infection. Biochem Biophys Res Commun 358(3):716-721

12. Hua R, Zhou Y, Wang Y, Hua Y, Tong G (2004) Identification of two antigenic epitopes on SARS-CoV spike protein. Biochem Biophys Res Commun 319(3):929-935

13. Prompetchara E, Ketloy C, Tharakhet K et al (2021) DNA vaccine candidate encoding SARS-CoV-2 spike proteins elicited potent humoral and Th1 cell-mediated immune responses in mice. PLoS ONE 16(3):e0248007

14. Tian X, Li C, Huang A et al (2020) Potent binding of 2019 novel coronavirus spike protein by a SARS coronavirus-specific human monoclonal antibody. Emerg Microbes Infect 9(1):382-385
15. McBride R, Van Zyl M, Fielding BC (2014) The coronavirus nucleocapsid is a multifunctional protein. Viruses 6(8):2991-3018

16. Chow SCS, Ho CYS, Tam TTY et al (2006) Specific epitopes of the structural and hypothetical proteins elicit variable humoral responses in SARS patients. J Clin Pathol 59(5):468-476

17. Che $X-Y$, Hao W, Wang $Y$ et al (2004) Nucleocapsid protein as early diagnostic marker for SARS. Emerg Infect Dis 10(11):1947

18. Grifoni A, Sidney J, Zhang Y, Scheuermann RH, Peters B, Sette A (2020) A sequence homology and bioinformatic approach can predict candidate targets for immune responses to SARS-CoV-2. Cell Host Microbe 27(4):671-680.e2

19. Cao B, Wang Y, Wen D et al (2020) A trial of Lopinavir-Ritonavir in adults hospitalized with severe covid-19. N Engl J Med 382(19):1787-1799

20. Chen L, Xiong J, Bao L, Shi Y (2020) Convalescent plasma as a potential therapy for COVID-19. Lancet Infect Dis 20(4):398-400

21. Raza S, Siddique K, Rabbani M et al (2019) In silico analysis of four structural proteins of aphthovirus serotypes revealed significant $B$ and $T$ cell epitopes. Microb Pathog 128:254-262

22. Tahir ul Qamar M, Shokat Z, Muneer I et al (2020) Multiepitope-based subunit vaccine design and evaluation against respiratory syncytial virus using reverse vaccinology approach. Vaccines 8(2):288

23. Ashfaq UA, Ahmed B (2016) De novo structural modeling and conserved epitopes prediction of Zika virus envelop protein for vaccine development. Viral Immunol 29(7):436-443

24. Ahmad B, Ashfaq UA, Rahman M, Masoud MS, Yousaf MZ (2019) Conserved $B$ and $T$ cell epitopes prediction of ebola virus glycoprotein for vaccine development: an immuno-informatics approach. Microb Pathog 132:243-253

25. Oany AR, Emran A-A, Jyoti TP (2014) Design of an epitope-based peptide vaccine against spike protein of human coronavirus: an in silico approach. Drug Des Dev Ther 8:1139

26. Grifoni A, Sidney J, Zhang Y, Scheuermann RH, Peters B, Sette A (2020) Candidate targets for immune responses to 2019-novel coronavirus (nCoV): Sequence homology- and bioinformatic-based predictions. SSRN Electron J 34:3931

27. Amer H, Alqahtani AS, Alaklobi F, Altayeb J, Memish ZA (2018) Healthcare worker exposure to Middle East respiratory syndrome coronavirus (MERS(oV): revision of screening strategies urgently needed. Int J Infect Dis 71:113-116

28. Tahir ul Qamar M, Shahid F, Aslam S et al (2020) Reverse vaccinology assisted designing of multiepitope-based subunit vaccine against SARSCoV-2. Infect Dis Poverty 9(1):132

29. Larsen JEP, Lund O, Nielsen M (2006) Improved method for predicting linear B-cell epitopes. Immunome Res 2(1):1-7

30. Reynisson B, Barra C, Kaabinejadian S, Hildebrand WH, Peters B, Nielsen M (2020) Improved prediction of MHC II antigen presentation through integration and motif deconvolution of mass spectrometry MHC eluted ligand data. J Proteome Res 19(6):2304-2315

31. Dimitrov I, Naneva L, Doytchinova I, Bangov I (2014) AllergenFP: allergenicity prediction by descriptor fingerprints. Bioinformatics 30(6):846-851

32. Magnan CN, Zeller M, Kayala MA et al (2010) High-throughput prediction of protein antigenicity using protein microarray data. Bioinformatics 26(23):2936-2943

33. Doytchinova IA, Flower DR (2007) VaxiJen: a server for prediction of protective antigens, tumour antigens and subunit vaccines. BMC Bioinform 8(1):4

34. Magnan CN, Randall A, Baldi P (2009) SOLpro: accurate sequence-based prediction of protein solubility. Bioinformatics 25(17):2200-2207

35. Gasteiger E, Hoogland C, Gattiker A et al (2005) Protein identification and analysis tools on the ExPASy server. In: Walker JM (ed) The proteomics protocols handbook. Humana Press, Totowa, pp 571-607

36. Colovos C, Yeates TO (1993) Verification of protein structures: patterns of nonbonded atomic interactions. Protein Sci 2(9):1511-1519

37. Laskowski RA, MacArthur MW, Moss DS, Thornton JM (1993) PROCHECK: a program to check the stereochemical quality of protein structures. J Appl Crystallogr 26(2):283-291

38. Wiederstein M, Sippl MJ (2007) ProSA-web: interactive web service for the recognition of errors in three-dimensional structures of proteins. Nucleic Acids Res 35(Web Server):W407-W410 
39. Heo L, Park H, Seok C (2013) GalaxyRefine: protein structure refinement driven by side-chain repacking. Nucleic Acids Res 41(W1):W384-W388

40. Madeira F, Park YM, Lee J et al (2019) The EMBL-EBI search and sequence analysis tools APIs in 2019. Nucleic Acids Res 47(W1):W636-W641

41. Grote A, Hiller K, Scheer M et al (2005) JCat: a novel tool to adapt codon usage of a target gene to its potential expression host. Nucleic Acids Res 33(Web Server):W526-W531

42. Vajda S, Yueh C, Beglov D et al (2017) New additions to the Clus Pro server motivated by CAPRI. Proteins Struct Funct Bioinform 85(3):435-444

43. Sayed SB, Nain Z, Abdullah F et al (2019) Immunoinformatics-guided designing of peptide vaccine against Lassa virus with dynamic and immune simulation studies. Preprints

44. Pandey RK, Verma P, Sharma D, Bhatt TK, Sundar S, Prajapati VK (2016) High-throughput virtual screening and quantum mechanics approach to develop imipramine analogues as leads against trypanothione reductase of leishmania. Biomed Pharmacother 83:141-152

45. Awan FM, Obaid A, Ikram A, Janjua HA (2017) Mutation-structure-function relationship based integrated strategy reveals the potential impact of deleterious missense mutations in autophagy related proteins on hepatocellular carcinoma (HCC): a comprehensive informatics approach. Int J Mol Sci 18(1):139

46. López-Blanco JR, Aliaga Jl, Quintana-Ortí ES, Chacón P (2014) iMODS: internal coordinates normal mode analysis server. Nucleic Acids Res 42(W1):W271-W276

47. Rapin N, Lund O, Bernaschi M, Castiglione F (2010) Computational immunology meets bioinformatics: the use of prediction tools for molecular binding in the simulation of the immune system. PLoS ONE 5(4):e9862

48. Castiglione F, Mantile F, De Berardinis P, Prisco A (2012) How the interval between prime and boost injection affects the immune response in a computational model of the immune system. Comput Math Methods Med 2012:1-9

49. Chauhan V, Singh MP (2020) Immuno-informatics approach to design a multi-epitope vaccine to combat cytomegalovirus infection. Eur J Pharm Sci 147:105279

50. Tahir-ul-Qamar M, Rehman A, Tusleem K et al (2020) Designing of a next generation multiepitope based vaccine (MEV) against SARS-COV-2: immunoinformatics and in silico approaches. PLOS ONE 15(12):e0244176

51. Khatoon N, Pandey RK, Prajapati VK (2017) Exploring Leishmania secretory proteins to design $B$ and $T$ cell multi-epitope subunit vaccine using immunoinformatics approach. Sci Rep 7(1):1-12

52. Laskowski RA (2009) PDBsum new things. Nucleic Acids Res 37(Database):D355-D359

53. Walls AC, Park Y-J, Tortorici MA, Wall A, McGuire AT, Veesler D (2020) Structure, function, and antigenicity of the SARS-CoV-2 spike glycoprotein. Cell 181(2):281-292.e6

54. Amanat F, Krammer F (2020) SARS-CoV-2 vaccines: status report. Immunity 52(4):583-589

55. Kumar J, Qureshi R, Sagurthi SR, Qureshi IA (2021) Designing of nucleocapsid protein based novel multi-epitope vaccine against SARS-COV-2 using immunoinformatics approach. Int J Pept Res Ther 27(2):941-956

56. Fournillier A, Dupeyrot P, Martin P et al (2006) Primary and memory T cell responses induced by hepatitis $C$ virus multiepitope long peptides. Vaccine 24(16):3153-3164

57. Mohabatkar H (2007) Prediction of epitopes and structural properties of Iranian HPV-16 E6 by bioinformatics methods. Asian Pac J Cancer Prev 8(4):602-606

58. Fung TS, Liu DX (2018) Post-translational modifications of coronavirus proteins: roles and function. Future Virol 13(6):405-430

59. Shajahan A, Supekar NT, Gleinich AS, Azadi P (2020) Deducing the N-and O-glycosylation profile of the spike protein of novel coronavirus SARSCoV-2. Glycobiology 30(12):981-988

60. Walls AC, Xiong X, Park Y-J et al (2019) Unexpected receptor functional mimicry elucidates activation of coronavirus fusion. Cell 176(5):1026-1039

61. Zhuang S, Tang L, Dai Y et al (2021) Bioinformatic prediction of immunodominant regions in spike protein for early diagnosis of the severe acute respiratory syndrome coronavirus 2 (SARS-CoV-2). PeerJ 9:e11232

62. Zarling AL, Ficarro SB, White FM, Shabanowitz J, Hunt DF, Engelhard VH (2000) Phosphorylated peptides are naturally processed and presented by major histocompatibility complex class I molecules in vivo. J Exp Med 192(12):1755-1762
63. Shamriz S, Ofoghi H, Moazami N (2016) Effect of linker length and residues on the structure and stability of a fusion protein with malaria vaccine application. Comput Biol Med 76:24-29

64. Oppenheim JJ, Biragyn A, Kwak LW, Yang D (2003) Roles of antimicrobial peptides such as defensins in innate and adaptive immunity. Ann Rheum Dis 62(suppl 2):ii17-ii21

65. Takamatsu N, Watanabe Y, Yanagi H, Meshi T, Shiba T, Okada Y (1990) Production of enkephalin in tobacco protoplasts using tobacco mosaic virus RNA vector. FEBS Lett 269(1):73-76

66. Barh D, Barve N, Gupta K et al (2013) Exoproteome and secretome derived broad spectrum novel drug and vaccine candidates in Vibrio cholerae targeted by Piper betel derived compounds. PLOS ONE 8(1): 52773

67. Burgess-Brown NA, Sharma S, Sobott F, Loenarz C, Oppermann U, Gileadi $\mathrm{O}$ (2008) Codon optimization can improve expression of human genes in Escherichia coli: a multi-gene study. Protein Expr Purif 59(1):94-102

\section{Publisher's Note}

Springer Nature remains neutral with regard to jurisdictional claims in published maps and institutional affiliations.

\section{Submit your manuscript to a SpringerOpen ${ }^{\circ}$ journal and benefit from:}

- Convenient online submission

- Rigorous peer review

- Open access: articles freely available online

- High visibility within the field

- Retaining the copyright to your article

Submit your next manuscript at $\mathbf{s p r i n g e r o p e n . c o m ~}$ 